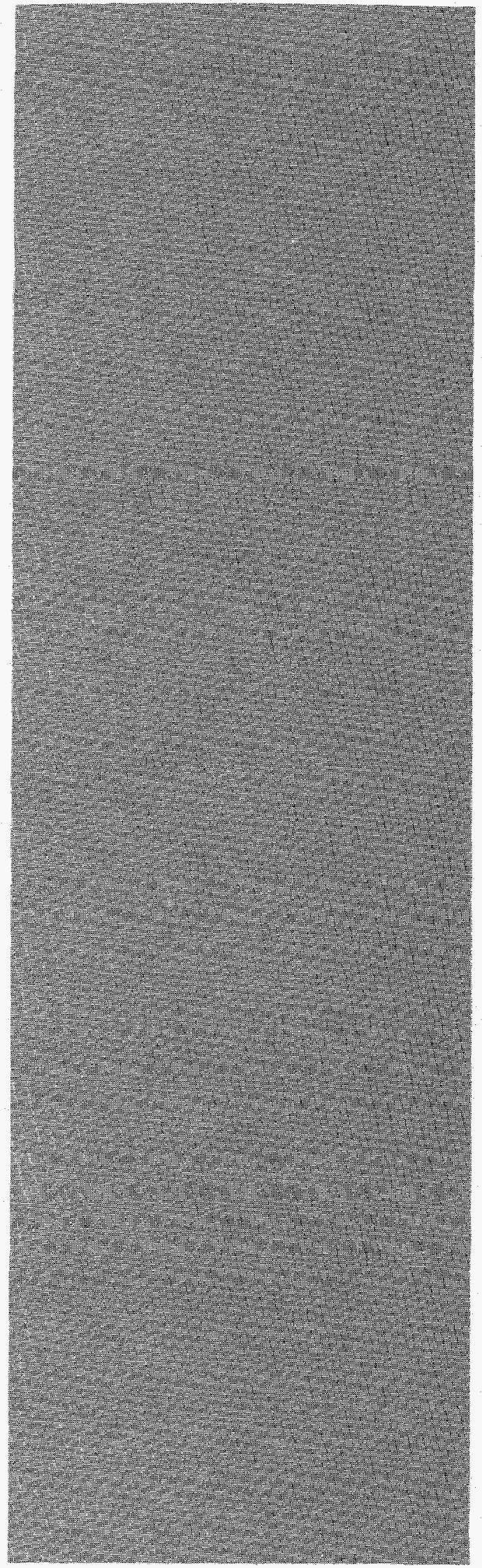

\title{
The NAVI-2: A Ruggedized
}

\section{Portable Radiation Analyzer}

\section{MASTER}

\section{Los Alamos}

NATIONAL LABORATOAY

Los Alamos National Laboratory is operated by the University of California for the United States Department of Energy under contract W-7405-ENG-36. 
Edited by Paul W. Henriksen, Group CIC-1

This work was supported by the U.S. Department of Energy, Office of Arms Control and Nonproliferation.

An Affirmative Action/Equal Opportunity Employer

This report was prepared as an account of work sponsored by an agency of the United States Government. Neither The Regents of the University of California, the United States Government nor any agency thereof, nor any of their employees, makes any warranty, express or implied, or assumes any legal liability or responsibility for the accuracy, completeness, or usefulness of any information, apparatus, product, or process disclosed, or represents that its use would not infringe privately owned rights. Reference herein to any specific commercial product, process, or service by trade name, trademark, manufacturer, or otherwise, does not necessarily constitute or imply its endorsement, recommendation, or favoring by The Regents of the University of California, the United States Government, or any agency thereof. The views and opinions of authors expressed herein do not necessarily state or reflect those of The Regents of the University of California, the United States Government, or any agency thereof. The Los Alamos National Laboratory strongly supports academic freedom and a researcher's right to publish; therefore, the Laboratory as an institution does not endorse. the viewpoint of a publication or guarantee its technical correctness. 
UC-700

Issued: January 1996

The NAVI-2: A Ruggedized

Portable Radiation Analyzer

W. S. Murray

K. B. Butterfield

C. M. Frankle 


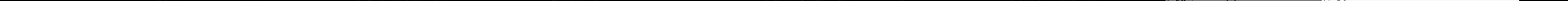


1. BACKGROUND 2

2. INTRODUCTION 4

2.1. Confirmatory Measurements 4

$\begin{array}{ll}\text { 2.2. Scanning } & 4\end{array}$

3. SYSTEM DESCRIPTION 6

3.1. Radiation Detection 6

3.1.1. Gamma Ray Detection 7

3.1.2. Neutron Detection 9

3.2. Electronics 10

3.2.1. Microcomputer 10

$\begin{array}{lr}\text { 3.2.2. Power Circuits } & 12 \\ & 13\end{array}$

3.2.3. Gross Counting 13

3.2.4. Pulse-Height Data Acquisition 13

3.3. Mechanical Packaging 14

4. SOFTWARE ANALYSIS ROUTINES

4.1. Boot-up Routine - GO 15

4.2. Data Histograms 16

4.3. Windowing 17

4.4. ROI Summation 17

$\begin{array}{ll}\text { 4.5. Plutonium Spectra } & 18\end{array}$

4.6. VERIFY 19

4.6.1. NON-SENSITIVE VERIFY 20

4.6.2. SENSITIVE VERIFY 20

4.6.3. PU PRESENT 21

4.7. Calibration $\quad 21$

4.7.1. Calibrate 23

5. USER DISPLAY 25

APPENDIX A: FLOW DIAGRAMS $\quad 27$

APPENDIX B: ELECTRICAL AND MECHANICAL SPECIFICATIONS SUMMARY 


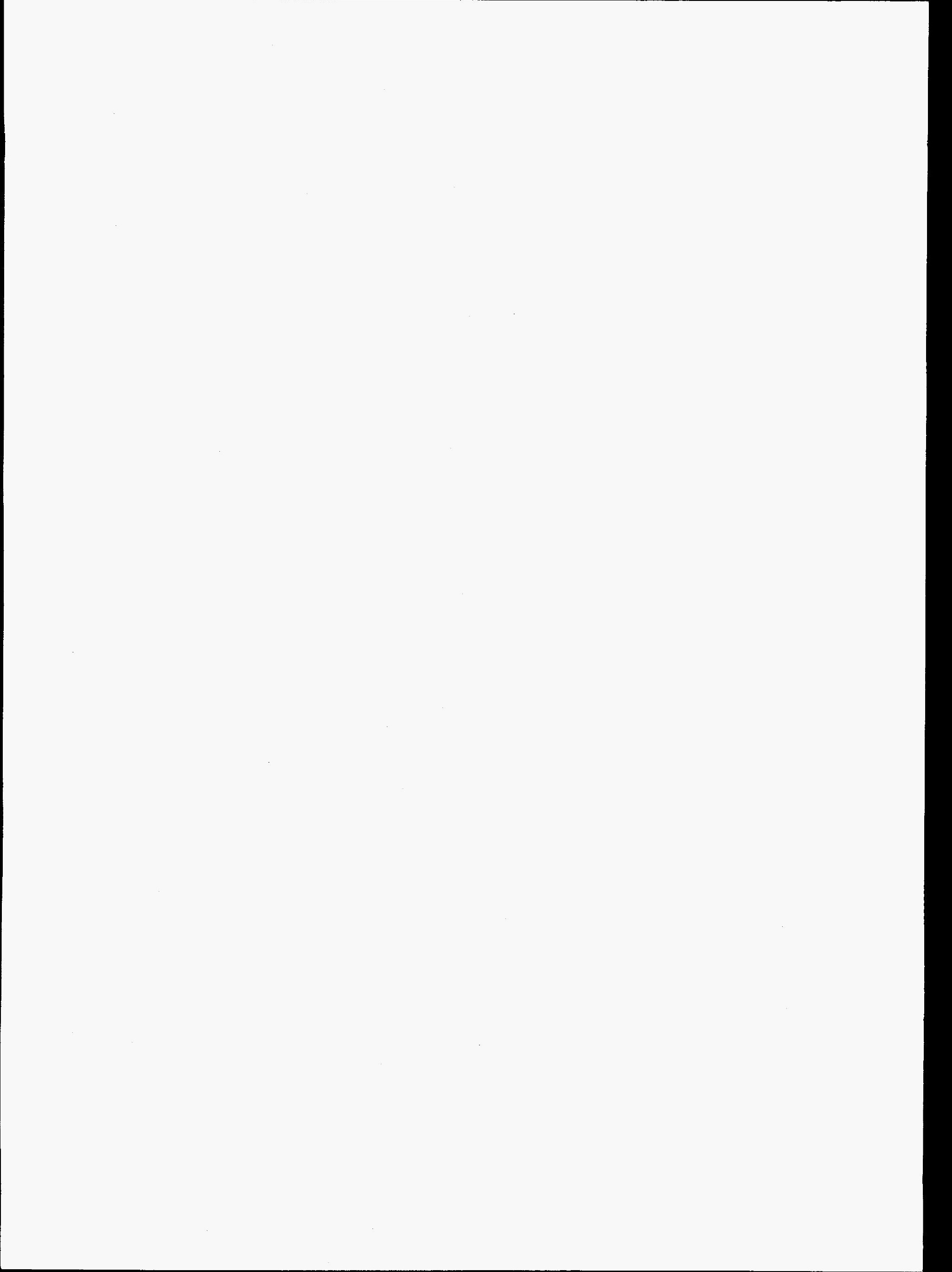


THE NAVI-2: A RUGGEDIZED PORTABLE RADIATION ANALYZER

by

W. S. Murray, K. B. Butterfield, and C. M. Frankle

\begin{abstract}
The NAVI-2 is a rugged, lightweight, and waterproof portable radiation analyzer developed by the Advanced Nuclear Technology group at Los Alamos National Laboratory. It was originally developed for a specific application: performing confirmatory measurements on plutonium removed from dismantled nuclear weapons as part of the O'Leary-Mikhailov mutual reciprocal inspections agreement. Since that time the hardware has stabilized into a mature package while several additional software packages have been developed. Now, in addition to the original software for performing confirmatory measurements, software is available that will allow the NAVI-2 to be used for scanning of extended sources, searching for hidden sources, and monitoring of items in a portal monitoring sense. This report will provide hardware and software details for owners and users of the NAVI-2.
\end{abstract}




\section{BACKGROUND}

In March 1994, U.S. Secretary of Energy Hazel O'Leary and Russian Minister of Atomic Energy Viktor Mikhailov announced an agreement whereby each side would host reciprocal inspections of each other's facilities containing plutonium removed from nuclear weapons. Shortly after the agreement was announced, the Department of Energy (DOE) convened a meeting of technical experts to consider what technical options would be available to implement the agreement. The DOE, with interagency concurrence, then developed a negotiating position where it was desired to obtain the technical resources to verify that a sealed storage container held a significant quantity of plutonium.

This position led the DOE to utilize a "Blue team - Red team" concept to find technologies that would be suitable for achieving the objective of verifying the presence of plutonium while also remaining unclassified. A number of both proprietary technologies developed at the national laboratories and commercial technologies were examined. The two teams concurred on the recommendation of two instruments, depending on the inspection scenario. If the scenario was such that measurements would be performed in a fully loaded storage vault, the recommended instrument was the RMS system from SNL. However, if the storage containers were to be brought out of the vault to a relatively low-background area, then the LANL NAVI-2 was recommended.

In the configuration that was recommended for the initial instrumentation demonstrations under the O'Leary-Mikhailov agreement, the NAVI-2 contained a low-resolution gamma-ray spectrometer. An onboard microprocessor recorded spectral data from the spectrometer and performed a real-time analysis of the data to ascertain the presence or absence of plutonium. Then, because spectral data on weapon components are considered sensitive, the instrument reported a result as "YES" or "NO" to the presence of plutonium and revealed no other data.

However, as the time for the demonstrations drew nearer, the DOE position changed from simple verification of the presence of plutonium to also include some form of mass measurement and some type of measurement that would reveal the shape of the object within the storage container. This presented added difficulties in that both the mass and shape of weapon components are classified. A less-than-satisfying but workable solution for the mass measurement was to have the neutron detectors in the NAVI-2 perform a socalled threshold measurement of the neutron flux. In this measurement the instrument 
would report a YES answer if the detected neutron count rate exceeded a predetermined threshold. However, to alleviate classification concerns, the preset threshold was set to correspond to approximately $100 \mathrm{~g}$ of U.S. weapons grade plutonium. A satisfactory solution for the shape measurement was not obtained in time for the initial instrumentation demonstrations and so low-energy radiography was demonstrated on an unclassified tantalum sphere.

After the initial instrumentation demonstrations, both sides agreed that the unclassified measurements demonstrated provided a very low level of confidence that the object within a storage container was indeed a weapon component. It was therefore agreed that if a so-called "Agreement for Cooperation" could be implemented between the sides to reciprocally share classified and sensitive information, then other, more revealing instrumentation techniques could be considered. After further negotiations the sides agreed to use limited high-resolution gamma-ray spectroscopy to determine plutonium isotopics and to use a slab neutron detector to record the total neutron count rate. This leaves shape determination as the final unresolved instrumentation issue.

The US has put forth the possibility of using the NAVI-2 equipped with a tight tungsten collimator to perform shape determination by gamma-ray scanning. In this mode the display is changed from YES to the net counts in the plutonium region-of-interest. This value is then recorded at each point on a spatial grid to allow the user to ascertain the shape of the plutonium within the container. For this particular application the neutron detectors are disabled, al though they could have been used in place of the slab detector.

The NAVI-2 then exists in two different versions, one in which sensitive data is displayed and one in which only non-sensitive data is displayed. The hardware is the same in all cases and any individual NAVI-2 can be changed from one version to the other by simply swapping the PROM chip containing the software. 


\section{INTRODUCTION}

The NAVI-2 portable radiation analyzer was designed by Los Alamos National Laboratory, Nonproliferation and International Security Division, Advanced Nuclear Technology Group (NIS-6) to provide sophisticated nuclear measurements in the field using a lightweight, battery operated, hand-held instrument. The system is packaged in a rugged, waterproof enclosure to withstand demanding conditions in the field. The microcomputer-based instrument detects both gamma rays and neutrons. A pulse height analyzer (PHA) is included to provide energy windowing of the gamma rays. The gamma detector may be collimated with various tungsten collimators for high-precision scanning. The NAVI- 2 can solve several radiation detection and analysis problems, depending on the particular version of software the NAVI-2 is running.

\subsection{Confirmatory Measurements}

VERIFY consists of identifying a radioactive source as plutonium. Plutonium emits both neutrons and gamma rays. The gamma rays are emitted at characteristic energies that can be used to identify the source as plutonium. The NAVI-2 discerns the energy of the gamma rays it detects and analyzes the resulting energy spectrum to determine if they are characteristic of plutonium. The presence or absence of neutrons increases the confidence of the results of the gamma-ray analysis because gamma rays can be easily shielded by intervening materials whereas the neutrons are difficult to shield. The programmable nature of the NAVI-2 simplifies tailoring the decision algorithms to any geometry or situation.

\subsection{Scanning}

The NAVI-2 is designed to scan radioactive sources. Scanning an object means using the radiation field of a source to create an image of the object to ascertain its geometry. The PHA performs energy "window" scans of a particular radioisotope by concentrating on the characteristic gamma rays, thereby excluding background and increasing the signal to noise ratio. The scan interval is programmable to allow for varying gamma-ray emission rates due to different amounts of intervening materials that may be present in a wide range of scenarios. 
A removable, tungsten collimator determines the spatial resolution of a scan. The standard NAVI-2 collimator (1.78 lbs.) is designed for a 45 degree opening angle and $80 \%$ attenuation at $400 \mathrm{keV}$ outside the acceptance cone. For applications requiring higher resolution, but not lighter weight, different collimators can be used by replacing the collimator end cap. When performing a line scan, the operator must determine the spacing (sampling interval) at which data is collected. The sampling interval should be uniform, planar, and half the spot size of the acceptance cone at the source position to avoid under or over sampling. Scanning is performed by pressing the "VERIFY" key at each desired location and manually recording the number of characteristic gamma rays observed. 


\section{SYSTEM DESCRIPTION}

The NAVI-2 is shown in Fig. 1. The gamma-ray detector is a ruggedized Na//PMT assembly commercially available from Bicron. Neutrons are detected by a matched pair of ${ }^{3} \mathrm{He}$ proportional counters commercially available from Reuter-Stokes. The detectors and three printed circuit board assemblies are housed in an anodized aluminum extrusion that is sealed on either end with precision machined covers. The snout on the front cover is threaded to permit the installation of a collimator. The rear cover houses the battery board which requires six lithium batteries for proper operation. A membrane keypad on the top provides single function push-button operation of the instrument. A 2-line by 16character LCD is visible through the membrane keypad and displays instrument results and status. D-rings on the side of the enclosure allow a number of strapping arrangements for easy carrying and operation.

The heart and soul of any nuclear instrument is the detectors and their electronics. These devices determine the inherent capability of the instrument. The brains of the instrument is the microcomputer. Through firmware, the microcomputer performs a variety of necessary tasks to manage the collection and analysis of the data available from the detectors. The brawn of the instrument resides with its mechanical packaging, which is engineered to withstand varying environmental conditions.

\subsection{Radiation Detection}

The nuclear detectors are both commercially available assemblies that are integrated into the NAVI-2 system. Each detector requires a high-voltage bias, which is necessary to amplify and collect the charge produced by nuclear interactions within the detectors. The charge is sensed by preamplifiers and shaping amplifiers that develop a voltage pulse large enough to be processed by digital electronics. The overall detector system is characterized over a wide temperature range $\left(-25^{\circ} \mathrm{C}\right.$ to $\left.75^{\circ} \mathrm{C}\right)$. The gain coefficients are stored in memory and the microcomputer is capable of adjusting the system gain to compensate for temperature swings, which can vary the gain as much as $25 \%$. 


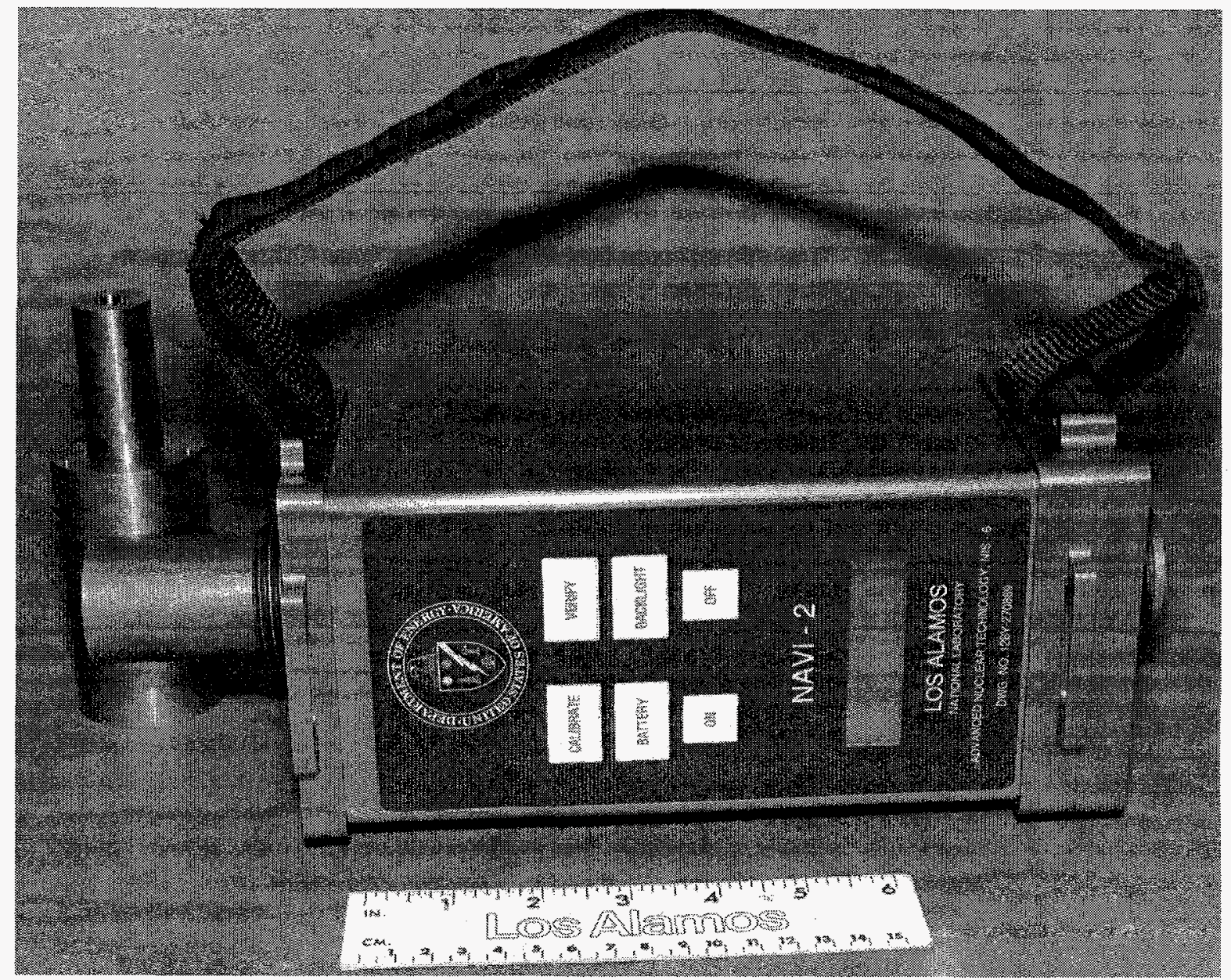

Fig. 1. A photograph of the NAVI-2.

\subsubsection{Gamma Ray Detection}

A scintillation detector operates when a gamma ray is absorbed by the active crystal, in this case sodium iodide $[\mathrm{NaI}(\mathrm{Tl})]$. This deposition of energy excites the outer shell of electrons. This electron state, however, is unstable and the electrons decay back to their original level, thus re-emitting the absorbed energy in the form of a flash of light. This flash of light travels through the crystal and is collected on the photocathode of the photomultiplier tube (PMT) where the photons are converted into free electrons. These electrons are collected and amplified by a series of dynodes in the PMT. The electrons are collected as a charge on the final dynode of the PMT where they are capacitively coupled into a charge sensitive preamplifier that outputs a voltage pulse. The amplitude of the resulting voltage pulse is proportional to the energy of the initial gamma ray. For a mono-energetic input, scintillators exhibit some spread in the distribution of light output, 
which is defined as the detector resolution. Light output also varies with temperature, which will show up as a gain shift in the energy spectrum.

$\mathrm{NaI}(\mathrm{Tl})$ is a medium resolution detector with limited inherent spectral identification capabilities. Resolving closely spaced isotopic complexes with $\mathrm{NaI}(\mathrm{Tl})$ is impossible but also unnecessary for simple radioisotope identification. The full width half maximum (FWHM) resolution of the detector is typically specified at the ${ }^{137} \mathrm{Cs}$ photopeak, 662 $\mathrm{keV}$. The FWHM is a ratio of the number of channels between the half maxima of the peak channel and the peak channel. The advantage of NaI(Tl) is the maturity of the technology and the ease of implementing it in a field instrument.

The gamma-ray detector is a Bicron Geoline series detector (model 1.125 MG2/1SSL-X). It is an extremely rugged detector assembly. The detector is drop tested to a height of one meter (compare that to the fact that most scientists would never consider dropping a scintillator coupled to a photomultiplier tube). The temperature gradient it can withstand is $3^{\circ} \mathrm{C} /$ minute (compare to $10^{\circ} \mathrm{C} /$ hour for laboratory-grade detectors). The outer jacket is stainless steel while the components are spring mounted in a proprietary potting compound. The $\mathrm{NaI}(\mathrm{Tl})$ scintillator is a right circular cylinder 2 in. long with a diameter of 1.125 in. The PMT is a ruggedized, high-temperature assembly from Hamamatsu. The gamma-ray detector exhibits a resolution slightly better than $9 \%$ FWHM at $662 \mathrm{keV}$.

To ruggedize the assembly, two major parameters were compromised. First, the resolution is $\sim 9 \% \mathrm{FWHM}$ at $662 \mathrm{keV}\left({ }^{137} \mathrm{Cs}\right)$. A laboratory grade $\mathrm{NaI}(\mathrm{Tl})$ detector would exhibit $\sim 6 \%$ resolution. In either case the resolution is not sufficient to resolve individual gamma rays in the complex of gamma rays near $400 \mathrm{keV}$ emitted by plutonium. This requires any analysis by $\mathrm{NaI}(\mathrm{Tl})$ to interpret the resulting broad peak that is formed due to the closely spaced peaks of plutonium (Fig. 2). Secondly, the stainless steel jacket and construction of the detector reduces the efficiency, especially at low energies. Because precise assay is not the point of verification measurements, the reduced efficiency is not detrimental to the operation of the instrument. 


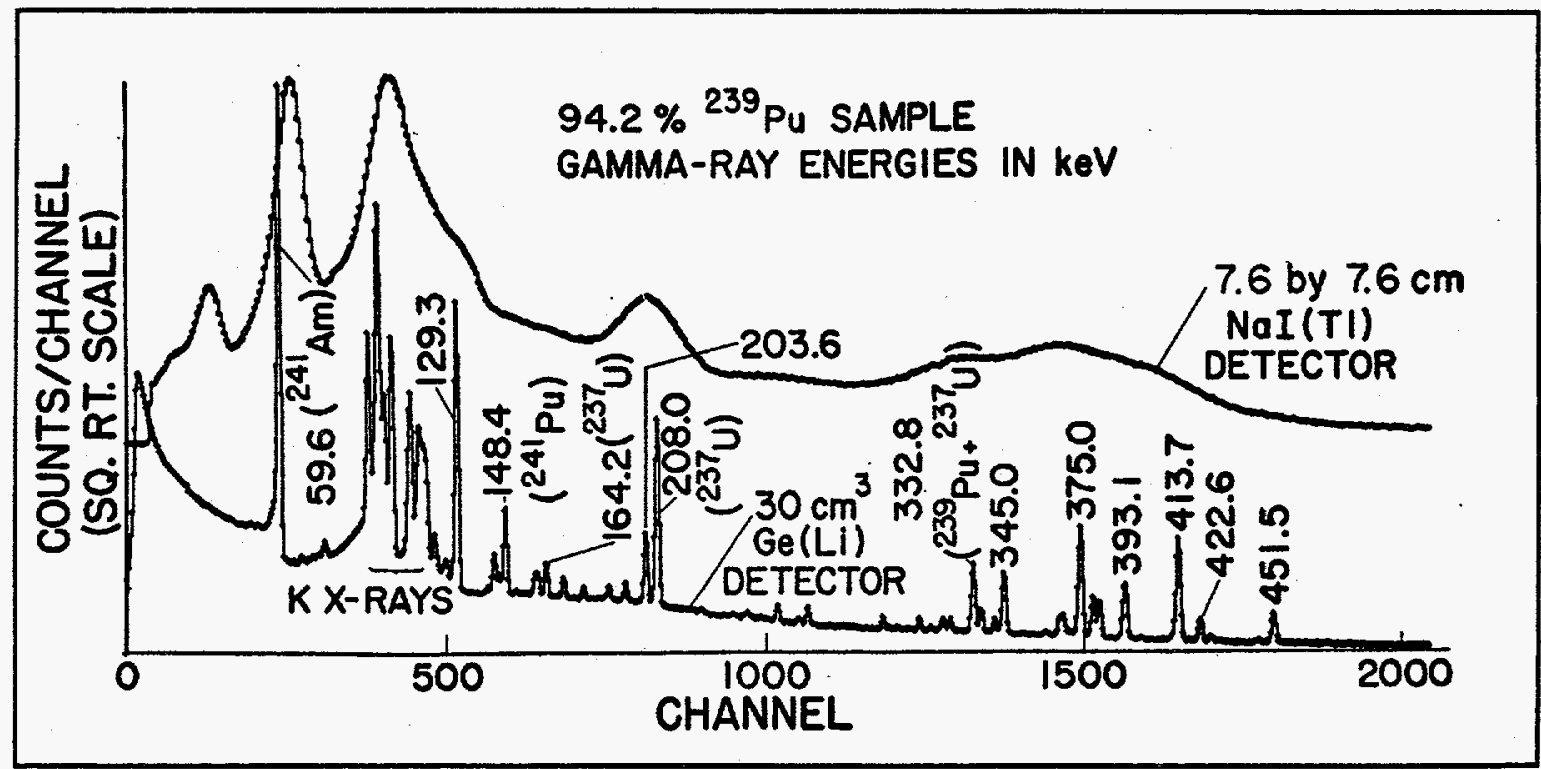

Fig. 2. Sample gamma-ray energies in keV of a sample of $94.2 \%{ }^{239} \mathrm{Pu}$.

The supporting electronics consist of a high-voltage power supply (HVPS) and a preamplifier/shaping amplifier. The HVPS (model Q-15, manufactured by Venus Scientific) provides bias to the PMT through an RC filter network. The preamplifier/shaping amplifier (model A-225, manufactured by Amptek, Inc.) is a hybrid integrated circuit (IC) and is charge coupled to the PMT through a HV capacitor. The output of the A-225 is a unipolar shaped pulse with a rise time of $2 \mu \mathrm{sec}$ and width of 10 $\mu$ sec.

\subsubsection{Neutron Detection}

Bare ${ }^{3} \mathrm{He}$ proportional counters (PC) preferentially detect thermal neutrons. Fission neutrons encompass a broad spectrum of energy and must be slowed down (moderated) before they can be detected by a ${ }^{3} \mathrm{He}$ detector. When a thermal neutron interacts with ${ }^{3} \mathrm{He}$ it is absorbed and the resulting nucleus splits into a proton and a triton. The electric field set up by the high-voltage bias accelerates the electrons to the anode of the PC and the positive ions to the wall. As the ions accelerate to the anode they collide with other atoms, freeing more electrons and increasing the free charge. The charge collected on the anode can be due to both the proton and the triton or either of the charges separately. This produces a thermal neutron spectrum, which has a thermal peak corresponding to collecting both charges and two distinct plateaus at one-fourth (proton only) and three 
fourths (triton only) of the peak energy. Charge collected below the one-quarter point is due to either noise or gamma rays. These events are filtered out by the low-level discriminator because they do not represent neutron interactions.

The capacitance of the proportional counter depends upon its cylindrical geometry and center anode wire. Mechanical vibrations cause the center wire to flutter, causing slight changes in the device capacitance. Due to the constant applied bias of $1700 \mathrm{VDC}$, these vibrations cause charge transfers, which appear to the electronics as neutron signals. Sharp blows to the case can cause these false signals, which will be evident by extremely high neutron count rates. False signals can also arise if the high-voltage components are stressed or have any stray capacitance to electrical ground in the form of condensate or some other contaminant.

The neutron detector is a pair of matched ${ }^{3} \mathrm{He}$ proportional counters (model RS-P4-0404214, manufactured by Reuter-Stokes). The active volume is a right cylinder 0.5 in. in diameter and 4 in. long. A 0.5 -in.-thick polyethylene annulus and a fill pressure of 20 atmospheres maximizes thermal neutron capture in a small lightweight package. Added efficiency can be gained by externally mounting a slab of polyethylene along the bottom of the instrument. Proportional counters are inherently rugged packages and no effort has been expended to ruggedize them further.

The supporting electronics consist of a high-voltage power supply (HVPS) and a preamplifier/shaping amplifier. The HVPS (model Q-30, manufactured by Venus Scientific) provides bias to the PC through an RC filter network. The preamplifier/shaping amplifier (model A-225, manufactured by Amptek, Inc.) is a hybrid integrated circuit (IC) and is charge coupled to the PC through an HV capacitor. The output of the A-225 is a unipolar shaped pulse with a rise time of $2 \mu \mathrm{sec}$ and width of 10 $\mu$ sec.

\subsection{Electronics}

\subsubsection{Microcomputer}

The electronics are matched to the detectors to make the most out of the data that is available. A block diagram of the electronics is shown in Fig. 3. A low-power 8-bit 
microcomputer (Motorola MC68HC11FN) is the engine for the NAVI-2 system. The microcomputer is affectionately dubbed the "FORTH engine," because it uses the FORTH programming language. The FORTH engine executes firmware that resides in a $32 \mathrm{~K}$ UV erasable EEROM. In addition, $24 \mathrm{~K}$ of battery-backed-up RAM is available for histogram storage and scratch areas for calculations. The 512 bytes of EEROM contain the interrupt vectors and system variables that must be maintained even when the batteries are changed.

The FORTH engine has five interface ports. Port $A$ is multipurpose and primarily used to count pulses from the LLDs. Port $B$ is output only and controls the application of power to the various subsystems. Port $\mathrm{C}$ is bi-directional and connects to the keypad. Port $\mathrm{D}$ provides a serial interface that is accessible through the battery compartment and provides remote access to the FORTH engine. This interface can be used to both control the instrument and retrieve archived data. Port $\mathrm{E}$ is a successive approximation 8-bit analog-to-digital converter, which is interfaced via buffer amplifiers to the system voltages to provide feedback on the health of the system. The interface to the spectrometer, LCD, and LLD level DAC are memory mapped, thus using the more efficient bus structure for data transfers.

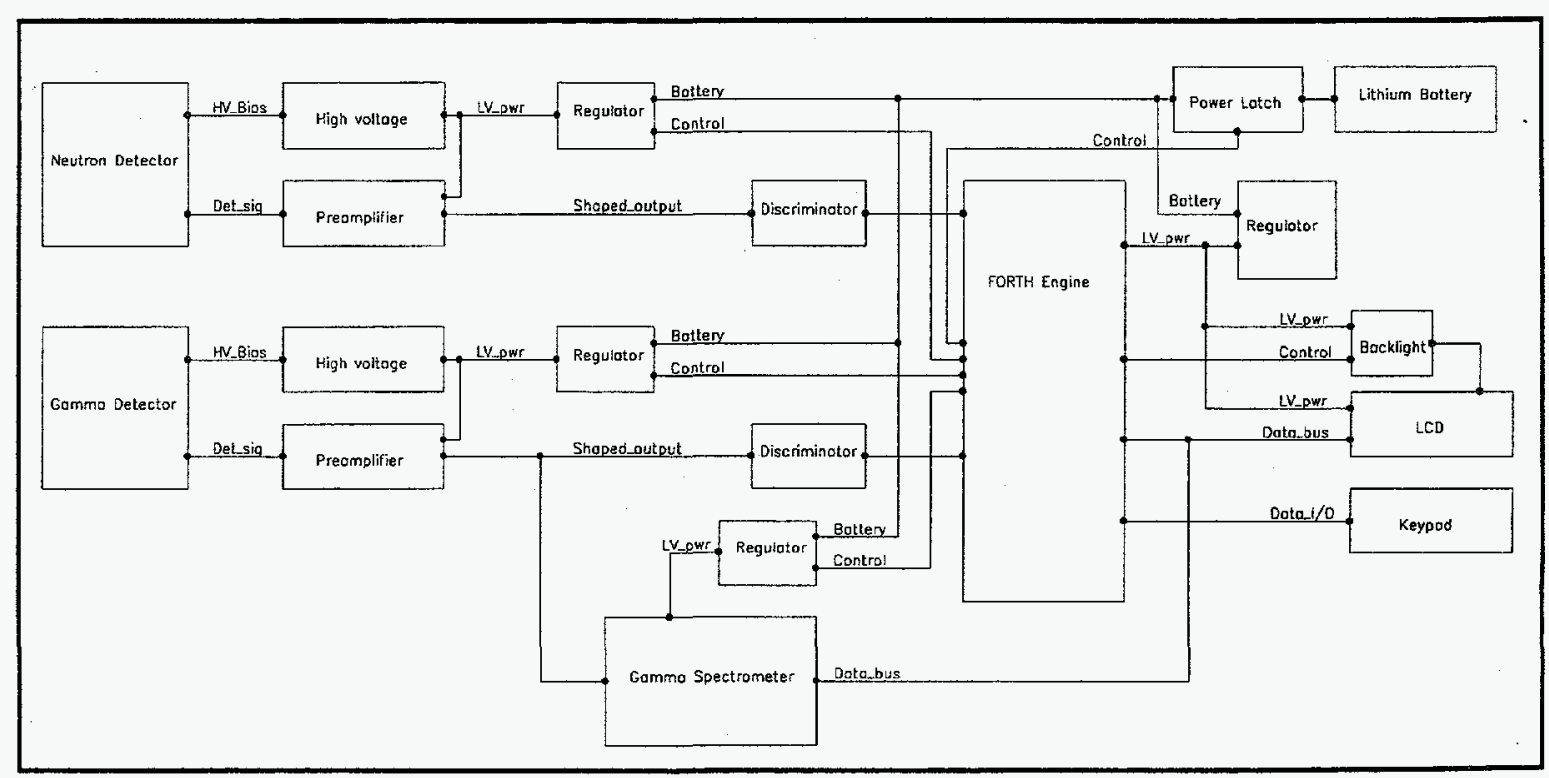

Fig. 3. Block diagram of the circuitry for the NAVI-2. 


\subsubsection{Power Circuits}

Power is supplied by lithium batteries. Three batteries in series nominally provide $9 \mathrm{~V}$ to the unit. The expected operating time is approximately 4 to 5 hours. An additional three batteries in parallel extends the lifetime to approximately 16 hours. When the ON button is pressed, the battery voltage is fed to a 5-V linear regulator, which supplies the FORTH engine. If the FORTH engine is successful in booting, then the battery power will be latched for a programmable amount of time (e.g., 30 seconds). If this time elapses before a function is selected, the FORTH engine will disable the latch thereby powering down the instrument. This saves batteries from inadvertent use and forgetful operators. The OFF button disconnects the primary batteries from the system. A second battery is employed to provide memory retention of the system RAM. This battery is designed to last approximately one year.

Power is managed by several voltage regulators that can be switched on or off depending upon the task the FORTH engine is trying to perform. The four power control circuits are as follows: LCD backlight power supply $(40 \mathrm{~mA})$, the neutron high-voltage power supply (12 mA), the gamma-ray high-voltage power supply (12 mA), and the gamma-ray $\mathrm{ADC}(22 \mathrm{~mA})$. The current savings is almost $50 \%$ when these modules are turned off. An icon in the first place of the LCD display indicates whether the backlight is on or off. During VERIFY, the neutrons, gamma rays, and PHA are turned on, thus enabling gross counting and spectral analysis. During SCAN, only the gamma rays and PHA are powered up. Some versions of the software do not take advantage of the power saving features if the programmed operation involves frequent switching on and off of the power supplies.

The microcomputer monitors the battery voltages along with the low-voltage feed to the neutron and gamma high-voltage power supplies. The system will respond with two voltage error messages: LOW BAT and NO HIGH VOLTAGE. A third level, NO BAT will result in the NAVI-2 shutting off automatically. The LOW BAT threshold is set approximately $1 / 4$ volt above the voltage necessary to operate the neutron HVPS because the low-voltage feed to the neutron HVPS is greater than the low-voltage feed to the gamma-ray HVPS. The NO HIGH VOLTAGE levels are set $60 \mathrm{mV}$ below the required level to operate the neutron HVPS and gamma ray HVPS respectively. The NO BAT threshold is set $1 / 4$ volt below the voltage required to operate the gamma rays. When the NO HIGH VOLTAGE level is reached, regulation to the HVPS is eliminated and the 
regulators saturate, draining the batteries even faster. Lack of regulation voids any energy calibration for the gamma-ray spectrometer because the system gain is highly dependent upon the PMT high-voltage bias.

\subsubsection{Gross Counting}

Gross counting indicates the number of gamma rays/neutrons detected without regard to energy. The absolute efficiency of the detectors is defined as the fraction of events counted to the total number of events occurring into $4 \pi$. The mass of an unshielded source can be estimated from the gross counts detected. Estimating mass from gamma ray gross counting suffers from a significant error term due to intrinsic shielding of the material itself or external materials. Mass estimates based on neutron gross counts are more precise but suffer from multiplication driven by geometric configurations of the fissile material and scatter if the neutrons are counted in a place where there is significant reflection of the neutrons. Whereas the efficiency of the detector can provide valuable insight into the minimum mass of radioactive material present, there are significant error terms present in assaying unknown material in unknown geometries.

Low-level discrimination (LLD) is the method used to exclude electronic noise and unwanted low-level signals from being counted as nuclear events. For neutrons, the LLD is set in a distinct valley between the one-fourth plateau (due to proton charge collection) and the electronic/gamma noise. This ensures counting all events due to neutrons but not gamma rays or noise. For the gamma rays, the LLD is set to provide a $60-\mathrm{keV}$ threshold. This is near the limit for low-energy response and the electronic noise for a $\mathrm{NaI}(\mathrm{Tl})$ based system.

The linear signals from the gamma and neutron shaping amplifiers are fed into a voltage comparator (LM393) where low-level discrimination is performed. The voltage (energy) thresholds are set by software through a buffered digital to analog converter (DAC). The digital signals are routed to the microcomputer where they are counted as a single event.

\subsubsection{Pulse-Height Data Acquisition}

The gamma-ray spectrometer consists of a high-speed buffer amplifier that drives the input to a flash analog-to digital converter (FADC) and high-speed discriminator. The discriminator feeds a pair of one-shots that generate a 50-nsec pulse that is the convert- 
clock signal for the FADC. The sampling scheme is nontraditional, in that the conversion is made $2 \mu \mathrm{sec}$ from the firing of the discriminator, which is approximately the rise time of the shaped output pulse from the preamplifier. For the majority of pulses, the conversion occurs at the peak and is a direct indication of the energy deposited in the detector. For the other pulses, a sampling error exists, which is corrected by a three-point quadratic energy calibration, performed in software. Interestingly, examination of the second-order term indicates that the sampling error is actually negligible. The resolution of the FADC is 8 bits $\pm 1 / 2$ LSB. This gives a 256-channel spectrum but there is considerable channel to channel deviation resulting in an effective 7-bit or 128-channel spectrum. The full-scale energy is nominally set at $1.6 \mathrm{MeV}$, which gives approximately $6 \mathrm{keV} / \mathrm{channel}$. At ${ }^{137} \mathrm{Cs}$ a $9 \%$ detector will result in about 10 channels FWHM.

When a pulse is converted by the FADC, an interrupt is generated and serviced by the FORTH engine. The FORTH engine's interrupt latency is $\sim 12 \mu \mathrm{sec}$, after which it fetches the FADC data and increments the proper memory location in a data array called HIST. When an analysis is performed, HIST is copied and stored in a working array called DATA. This allows uninterrupted data collection to proceed while computations are performed. Memory is available to archive up to 14 histograms in a circular fashion (i.e., when the fourteenth histogram is saved the next histogram will overwrite the first histogram).

\subsection{Mechanical Packaging}

The mechanical package is designed to be rugged, lightweight, and waterproof. It consists of a 6061 aluminum extrusion capped on either end with precision machined aluminum caps and laser-cut neoprene gaskets. THE electronics and detector assembly is easily removed by removing the retaining screws on the rear end cap and sliding the package out. The keypad must be disconnected before the assembly is completely free. The front end cap has a threaded detector snout, which can accommodate a tungsten collimator for some applications. The rear end cap separates into two halves via four thumb screws. This provides access to the batteries and the serial port. 


\section{SOFTWARE ANALYSIS ROUTINES}

This section describes the algorithms used to collect and analyze data from the radiation detectors. In most respects NAVI-2 is a hardware platform much like the IBM PC. What defines a particular implementation of NAVI-2 is the software. Much software has been written for the NAVI-2 platform. Some routines that are available but not currently implemented are portal monitor functions such as moving averages or the sequential probability ratio test, long-term monitoring and recording, searching functions such as rate meters or programmable rate meters, and scanning functions to produce images of radioactive sources. Displays that can be used are LEDs, LCD characters, or large graphic arrays capable of displaying images or spectra. Inputs can be from switches, single function keypads, or remote terminals.

Regardless of the suite of software options one may find loaded on the NAVI-2 platform, many of the routines are the same. The basic routines are described below. For each of the software descriptions there is an accompanying flow diagram that explains the software actions that are taken. Please refer to them as you read the descriptions.

Flow Diagram 1.

Flow Diagram 2.

Flow Diagram 3A.

Flow Diagram 3B.

Flow Diagram 4.
GO

CALIBRATE

NON-SENSITIVE VERIFY

SENSITIVE VERIFY

PU PRESENT

\subsection{Boot-up Routine - GO}

This section describes the boot-up routine GO, which is executed upon power-up. Refer to Flow Diagram 1.

When power is applied to the NAVI-2, the FORTH engine searches memory at every $1 \mathrm{k}$ boundary for an autostart vector, which is indicated by the hex number A55A. The next two bytes after $A 55 \mathrm{~A}$ point to an executable routine, in this case $\mathrm{GO}$, that the processor jumps to and begins executing. GO initializes the system and begins to check for a timeout that will result in a power down or a key press. If a key press is sensed, then the 
MENU NAVI is invoked. MENU NAVI decodes the key either from the serial port or the keypad. If the key is "0," CALIBRATE is invoked; if " 1 ," VERIFY is invoked; if "2," BATTERY is invoked; if "A," SYSTEM is invoked; or if "B," STATUS is invoked. Any other key is ignored and MENU NAVI awaits the next key press. " $A$ " and " $B$ " may only be received from a remote terminal. Once the MENU NAVI is invoked, automatic POWER DOWN can only be achieved if CALIBRATE or VERIFY is invoked and finishes without any subsequent keys being pressed. This is illustrated in the flow charts detailing these functions.

\subsection{Data Histograms}

Pulses from the gamma-ray detector are digitized and histogrammed. The FADC is an 8bit converter, which results in 256 channels that can be used to describe the pulse height distribution of the incoming pulses. Although the converter has 8-bits, quantization error degrades the resolution to effectively 7 bits or 128 channels. All routines use the 256 channels rather than binning the ADC results by 2 . A software filtering function can be utilized to make the data appear smoother.

The histogram memory area is 512 bytes long, accommodating 16 bits per channel or 65,536 pulses per channel. Overflow conditions create unusable histograms and, when detected, cause data collection to cease. Several areas in memory are set aside for histograms. Pulse height data coming directly from the gamma-ray detector is stored in an array named HIST. Data can be transferred from HIST to an array named DATA for analysis. No analysis is ever performed on the HIST array because incoming data may change before the analysis is complete. Therefore before any analysis is performed, the data in HIST is copied to DATA. Additional arrays exist to archive spectra for later retrieval. These stored spectra can be loaded back into DATA for subsequent analysis.

Data is stored into HIST on an interrupt basis. A pulse from the gamma-ray detector causes an interrupt, which is serviced by the microcomputer by reading the ADC, doubling the value, which is then used as a vector into the array HIST, incrementing the memory location, and checking for overflows. The machine time to perform these functions is on the order of $30 \mu \mathrm{sec}$. If the rate of pulses coming in from the gamma-ray detector exceeds $\sim 30 \mathrm{kHz}$, then the microcomputer is totally tied up processing pulses and cannot service keypad requests, update the display, or copy incoming data to the 
DATA array for analysis. Because the arrival of nuclear events is not periodic, degradation of system performance can be noticed at count rates of around $\sim 20 \mathrm{kHz}$.

\subsection{Windowing}

Windowing is the term used for viewing or analyzing part of the total spectrum of gamma-ray events. Gamma rays emitted from radioactive material are unique in their energy and intensity, thus creating a fingerprint from which one can determine the isotopic composition. Whereas a $9 \% \mathrm{NaF}(\mathrm{Tl})$ detector is inadequate for precision assays, it can be used to detect the presence or absence of an isotope in many cases.

Windowing is used to zoom in on a region of interest (ROI) that contains the characteristic gamma ray that can be used to identify the isotope. Windows can be defined as energy regions such as $330 \mathrm{keV}$ to $450 \mathrm{keV}$, or as channel extents such as channel 34 to channel 75 . A calibration curve allows interchanging the two units.

\subsection{ROI Summation}

The ROI summation technique is the method used when a window is opened to determine if the gamma rays resulting from the decay of a radioisotope are present. When a window is opened, the background term for that window is calculated by expanding the window on either side by five channels and using the average number of counts in these regions to provide endpoints for a straight-line approximation. This approximation is subtracted from the total number of counts in the ROI to give the net counts in the region above background. The net counts can be negative, which usually indicates the absence of any above-background gamma-ray activity in that region. Poor choice of the background defining regions can also lead to negative net counts, but this is due to poor judgment in the definition of the regions, poor calibration, or interfering sources that essentially are masking the characteristic gamma-rays of a particular isotope.

An example of the straight-line estimation for background $B$ and the variance $\sigma^{2}(B)$, when the background ROIs are placed symmetrically about the peak and are equally wide, is given by 


$$
B=\frac{N_{p}}{2 N_{b}}\left(B_{H}+B_{L}\right)
$$

and

$$
\begin{aligned}
& \sigma_{B}^{2}=\left(\frac{N_{p}}{2 N_{b}}\right)^{2}\left(B_{H}+B_{L}\right) \\
& \text { where } \\
& \qquad \begin{aligned}
B_{H} & =\text { the integral counts in the high-background ROI, } \\
B_{L} & =\text { the integral counts in the low-background ROI, } \\
N_{p} & =\text { the number of channels in the peak ROI, and, } \\
N_{b} & =\text { the number of channels in the background ROI. }
\end{aligned}
\end{aligned}
$$

The peak area $A$ is the difference of the integral of the peak ROI $P$ and the contribution from the background $B$ and can be described by

$$
A=P-B
$$

The estimated variance of the peak area $\left(\sigma_{A}^{2}\right)$ using the straight line background interpolation is

$$
\sigma_{A}^{2}=\sigma_{P}^{2}+\sigma_{B}^{2}=P+\sigma_{B}^{2}
$$

The standard deviation of the peak area $\sigma_{A}$ is used as an indicator if the integral counts in a region are above background. Although the threshold for declaring an isotope present by net counts above background is programmable, the typical value used is six standard deviations.

\subsection{Plutonium Spectra}

Specifically, for plutonium, the region between $330 \mathrm{keV}$ and $450 \mathrm{keV}$ is examined for net counts as explained in the previous section. A natural background spectrum always exhibits a negative slope in this region making it virtually impossible to come up with positive net counts without a source present. There are, however, other sources besides plutonium that can produce net counts in this region. Most notable is ${ }^{133} \mathrm{Ba}$ (which is used as a simulant in training). Barium-133 has a gamma ray at $356 \mathrm{keV}$ that is especially 
intense and will easily drive the net counts to six standard deviations above background in a short time. To guard against false positives and identify ${ }^{133} \mathrm{Ba}$ as the training simulant or spoofing agent, the plutonium window is separated into two halves and a ratio is computed of the net counts in both halves. The gamma rays emitted by plutonium are closely spaced in energy and are not fully resolved by a $9 \% \mathrm{NaI}(\mathrm{Tl})$ detector. Rather they create a broad complex of peaks that differs from a single peak such as from ${ }^{133} \mathrm{Ba}$. The energy from ${ }^{133} \mathrm{Ba}$ resides in the lower half of the energy window whereas the energy from plutonium is spread more evenly across the region. This allows for a ratio to be formed contrasting the number of counts in the lower half to the number of counts in the upper half. Barium-133 will have significantly more counts in the lower half while plutonium will be relatively even between the two halves. A factor of six is used as the threshold to discriminate between the simulant ${ }^{133} \mathrm{Ba}$ and plutonium.

This is not the only false positive that can occur. Compton edges from isotopes such as ${ }^{137} \mathrm{Cs}$ can cause net counts above background in the plutonium region. This is handled differently. The Compton edge is even flatter than the plutonium complex of peaks and the centroid tends to occur at higher energies. A second moment or variance test is done to discern the shape of a Compton edge and a complex of peaks. This test is not as robust as the ratio test but definitely quantifies the shape of the peak as very flat and probably not due to the emission of characteristic gamma rays.

\subsection{VERIFY}

There are two software versions of VERIFY described here. The first version to be described will be the non-sensitive version and the second will be the sensitive version. The non-sensitive version does not reveal any classified information, only a YES/NO to the question of plutonium presence and a threshold number of neutrons. The sensitive version reveals the number of gamma rays detected in the plutonium ROI for a given collection time. This information is deemed classified for weapon components. Many other routines are also available depending on the type of results that one may want to report. 


\subsubsection{NON-SENSITIVE VERIFY}

This section describes the actions taken by the microcomputer to perform a non-sensitive verify. Refer to Flow Diagram 3A.

The system is initialized to take data by turning on the ADC, enabling dead time calculations and setting the collection time. Data collection begins by resetting user flags and synchronizing the interrupt enable with the real time clock. Incoming pulses are digitized and histogrammed on an interrupt basis. During that time a software loop is checking for key presses, time conditions, and errors. Errors are reported to the screen, key presses cause the loop to abort, and timing conditions do one of two things. A minimum time is programmed before the histogrammed spectrum is checked for plutonium, usually 5 seconds. A maximum time is set to limit the collection to a reasonable value, such as 60 seconds. Either time can be programmed for a particular situation. Once the minimum time has elapsed, the spectrum is copied from the acquisition histogram, HIST, to the analysis histogram, DATA. The PU PRESENT routine determines if plutonium is present based upon some confidence factor, typically six sigma. If plutonium is not found, the loop begins over and the data is checked every time through the loop. If plutonium is found, the ADC is powered down and the system is essentially idle until the maximum time is reached. In this manner the operator has no information as to the counting statistics that lead to the determination of the presence of plutonium. When the maximum time is reached, the data is checked one last time and the results are reported as YES/NO. In some cases a neutron counter is installed and the neutron count rate is calculated and reported as YES/NO if the count rate exceeds a programmable threshold. Once the analysis is finished, a time-out period is invoked during which the user must press a key or the instrument will power itself down.

\subsubsection{SENSITIVE VERIFY}

This section describes actions taken by the microcomputer to perform a sensitive verify. Refer to Flow Diagram 3B.

The sensitive version is initialized in the same manner as the non-sensitive version and data is acquired in precisely the same manner. The end conditions and result reporting are the only differences in the routines. Data is taken until a preset time expires, then the net count in the plutonium ROI is calculated by PU PRESENT and reported. Once the 
analysis is finished, a time-out period is invoked during which the user must press a key or the instrument will power itself down.

\subsubsection{PU PRESENT}

This section describes actions taken by the microcomputer to perform an ROI summation to verify the presence of plutonium. Refer to Flow Diagram 4.

PU PRESENT is the actual routine that defines the window, calculates the net counts and the variance. For plutonium the window is defined from $330 \mathrm{keV}$ to $450 \mathrm{keV}$. The plutonium complex in this region will create a broad peak in the spectrum that can be compared to background. As long as there are no interfering background sources, this method is reliable in determining the presence of plutonium. It compares the standard deviation of the net counts above background to a programmable threshold level and determines by that if the lines from plutonium are present. If the six-sigma test is not met, NOT FOUND is reported. If the criteria are met, the net counts in the region are reported as $\mathrm{PU}$, unless the instrument has undergone an in situ calibration in which case a test is performed to determine if ${ }^{133} \mathrm{Ba}$ is present. If ${ }^{133} \mathrm{Ba}$ is found, then the counts are reported as BARIUM instead of PU.

\subsection{Calibration}

The detected gamma-ray energy is related to the channel number of the FADC by the calibration curve. During initial calibration, the operator must present three known gamma rays of differing energies and determine the FADC channel number that they are detected in. The FORTH engine takes this input and solves the quadratic equation that defines the curve the three points satisfy. The FORTH engine then fills a table of values relating each channel to an energy value. From this point forward the FORTH engine can work in energy units when discerning gamma rays.

A ${ }^{207} \mathrm{Bi}$ source (Fig. 4) is preferred for initial calibration of the NAVI-2 because it has three easily distinguished gamma rays or lines: $80 \mathrm{keV}, 570 \mathrm{keV}$ and $1064 \mathrm{keV}$. A number of other sources have been used for calibration including ${ }^{232} \mathrm{Th},{ }^{133} \mathrm{Ba},{ }^{137} \mathrm{Cs}$, and ${ }^{60} \mathrm{Co}$. Only ${ }^{232} \mathrm{Th}$ has enough lines to be used alone and then the highest energy line that falls within the full scale energy (FSE) of the FADC is $583 \mathrm{keV}$. That leaves some 
room at the high end for extrapolation errors. However, if plutonium is the only concern then the FSE could be halved $(800 \mathrm{keV})$ and ${ }^{232} \mathrm{Th}$ is a viable source whereas the ${ }^{207} \mathrm{Bi}$ would be short a line.

For auto-calibration initiated from the keypad, the operator must present three gamma rays to the NAVI-2, but in this instance the NAVI-2 will determine the peak channels, check that they are within acceptable values, store them, and recalibrate the instrument. It is important that the operator use the same three gamma rays that were used for the initial calibration or the procedure will fail. The algorithm allows a preset time (nominally 60 seconds) for sufficient statistics to build up so that the FORTH engine can find the peaks. Otherwise the procedure fails. The gamma-ray peaks found during auto calibration must also not deviate from the initial calibration more than $\pm 10 \%$ or the procedure will fail. If the unit is able to auto-calibrate, it is a very good functionality test of the hardware and software. If auto-calibration fails, then the unit must be serviced. This servicing involves using the serial port to determine which test the NAVI-2 did not satisfy and correcting the situation. Although the detector used is extremely stable as nuclear detectors go, calibration is desirable to assist high confidence in-field measurements.

A temperature check is performed to see if additional gain corrections to the default have to be made before attempting to calibrate. Characterizations of the temperature vs. gain curve indicate $5 \%$ variations between $15^{\circ} \mathrm{C}$ and $55^{\circ} \mathrm{C}$. No attempt to compensate is performed in this range. Outside this range a piecewise linear approximation to the gain curve is invoked to place the defaults in a more suitable range, thus increasing the likelihood of a successful calibration.

If a calibration source is not available, or the calibration fails, the default calibration will be used for the Verify analysis. However, the $\mathrm{Ba} / \mathrm{Pu}$ ratio and Compton tests will not be performed under default conditions. Default conditions may be acceptable if there is confidence that verification is being done on genuine items and no attempt to spoof is likely. 


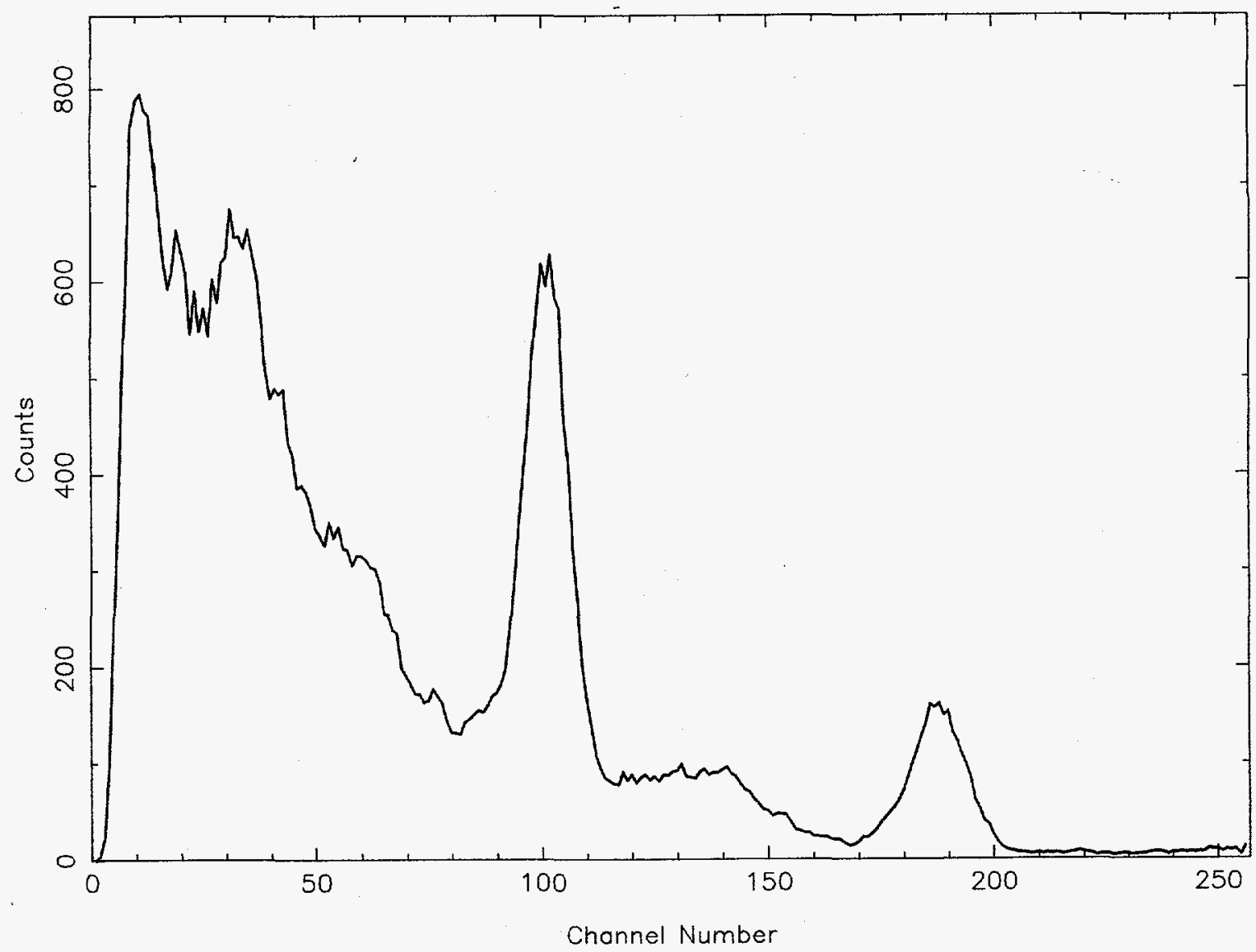

Fig. 4. Gamma-ray spectrum from a ${ }^{207} \mathrm{Bi}$ source.

\subsubsection{CALIBRATE}

This section describes the actions taken by the microcomputer when the CALIBRATE key is pressed. Refer to Flow Diagram 2.

CALIBRATE automatically checks and adjusts the energy calibration of the NAVI-2. Upon power up, the DEFAULT CALIBRATION is checked and verified. Gain drifts can cause the default calibration to be slightly in error. Generally, this isn't enough to cause the instrument to malfunction; however, in the interest of reliability the operator can calibrate immediately before a measurement to insure proper functioning. A successful calibration is a thorough test of all the instrument's abilities. The in situ calibration is erased and the DEFAULT restored when power is cycled. An error during calibration will also cause the DEFAULT values to be used. 
Specifically, calibrate begins by turning on the ADC power, enabling dead time calculations, and setting the programmable collection time. It checks the temperature, and if it is out of range, a compensation algorithm will adjust the default values. This gives the actual calibration a better chance to find the peaks. Once this initialization is done, data collection begins. Data is collected on an interrupt basis. At any time a key press will abort the process and the resulting calibration values will either be the default or temperature-compensated values. If the process goes uninterrupted by the user then the data is copied from HIST to DATA. Three separate ROIs are opened and analyzed for net counts greater than six sigma and centroid deviation less than $10 \%$ from the DEFAULT value. If each ROI can pass this test, then the new values are written, the calibration array is recalculated, and a calibration flag is set indicating a successful process. If any of the peaks are not found then the process stops, an ERROR is displayed, and either the DEFAULT or temperature-compensated values are then used.

The NAVI-2 waits for a programmable amount of time before auto-powering down for the next key press. If a key is sensed before this time-out period, it is decoded and the appropriate function invoked by MENU NAVI. 


\section{USER DISPLAY}

Under U.S. classification guidelines both the mass and the shape of a weapon component are classified. As a consequence, the options for displaying data by the NAVI-2 in a nonsensitive mode are quite limited. In addition, all sensitive data obtained by the NAVI-2 is stored in volatile memory such that no data remain after the power is turned off. Therefore, in the non-sensitive mode all results are reported by the NAVI-2 as YES or NO.

For the gamma-ray detector, the NAVI-2 is set up to always count for 60 seconds so that quantitative results cannot be obtained by watching when an affirmative decision is reached. However, during the 60 -second interval, the NAVI-2 is continuously analyzing the incoming gamma-ray spectral data. As soon as an affirmative decision is reached (the five standard deviation test is passed) data acquisition is stopped. The barium ratio test is then performed. If this test is successfully passed, then at the end of the 60 -second interval the upper line of the display changes to PU: YES. Under any result the display changes to PU: NO.

The neutron detectors follow the same count rate scheme as the gamma-ray detectors with no result being reported until the end of the 60 -second interval. However, during that time the neutron detectors are continuously acquiring data. At the end of the interval the neutron count rate for the interval is computed. If the calculated rate exceeds the preprogrammed threshold then the lower line of the display changes to NU: YES. Otherwise the lower line of the display changes to NU: NO.

In the sensitive data display mode, it is assumed that while sensitive or classified data can be displayed, it may still be undesirable to display the complete gamma-ray energy spectrum. Because numerical data may now be displayed, the NAVI-2 may now be used in two ways. First, one may perform the same type of verify operation as in the nonsensitive mode. Second, by installing a collimator around the gamma-ray detector, one may now perform spatial scans to determine the extent of the material.

As in the non-sensitive mode, the NAVI-2 still uses a 60 -second count interval. At the end of this interval the net counts in the plutonium region of interest are displayed on the first line of the display if the five-standard-deviation test and the barium ratio test have both been passed. The display line should look like this: PU: 12847. If the barium ratio 
test is not passed, then the display will report the net counts in the barium region of interest: $\mathrm{Ba}: 3856$. Finally, if the five-standard-deviation test is not passed, the display will indicate as follows: PU: Not found. The lower line of the display will indicate the neutron count rate as in the following: NU: 1.33. Taken together these two numerical results will allow the operator to compute both an upper and lower bound on the mass of plutonium present.

If the operator attaches a collimator to the gamma-ray detector and repeats the verification procedure at multiple points, a spatial map of the gamma-ray emissions may be built up. It should be noted that the shape of the collimator has a direct bearing on the spatial resolution that can be achieved by this procedure. Also, the neutron count rates are relatively useless in such a procedure due to the high average number of scatterings that neutrons undergo before they are detected. The spatial map of gamma-ray emissions can be utilized to determine the basic shape of the object being scanned if the available collimator has sufficient precision. 
Appendix A: Flow Diagrams

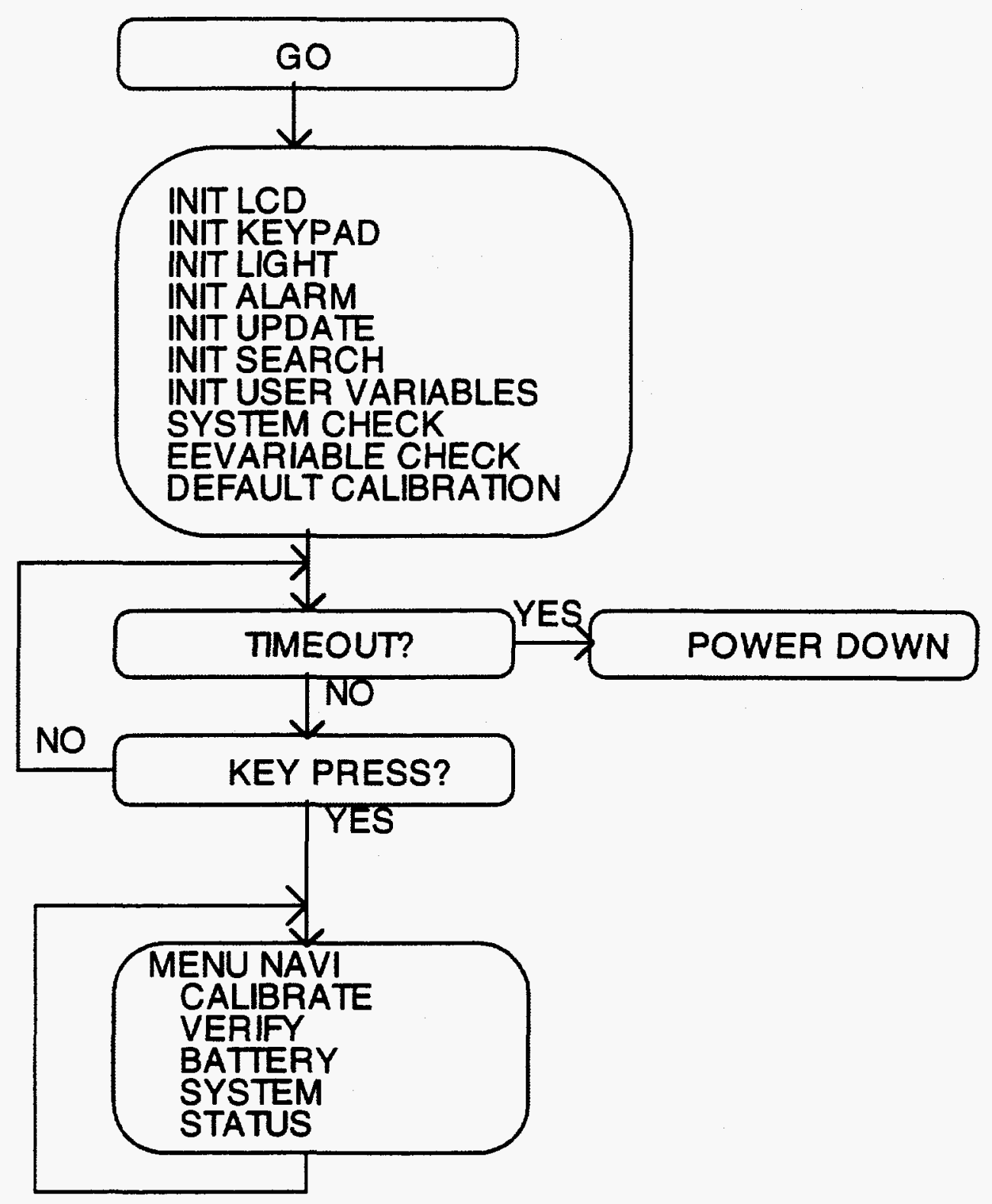

FLOW DIAGRAM 1 


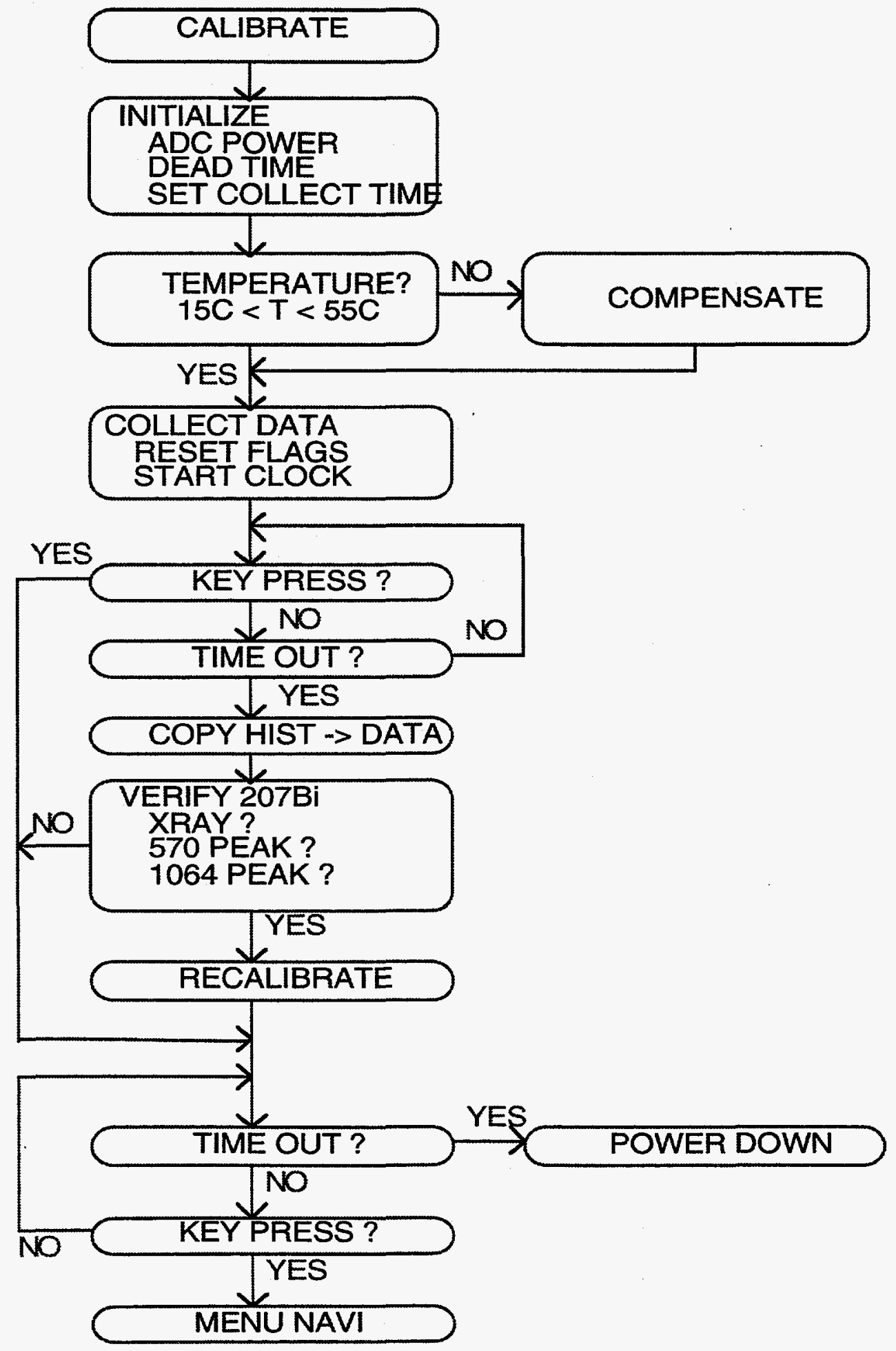

FLOW DIAGRAM 2 


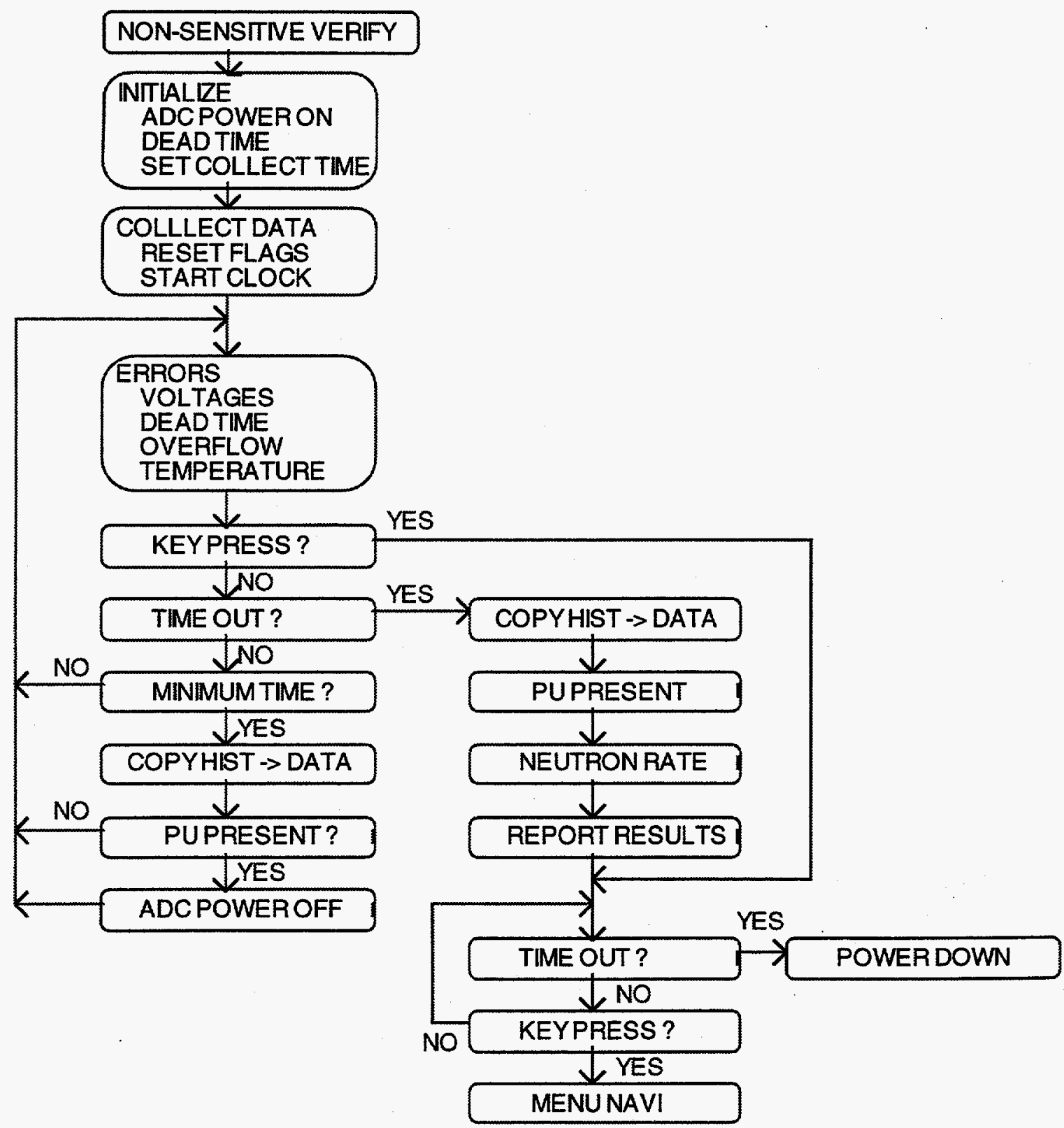

FLOW DIAGRAM 3A. 


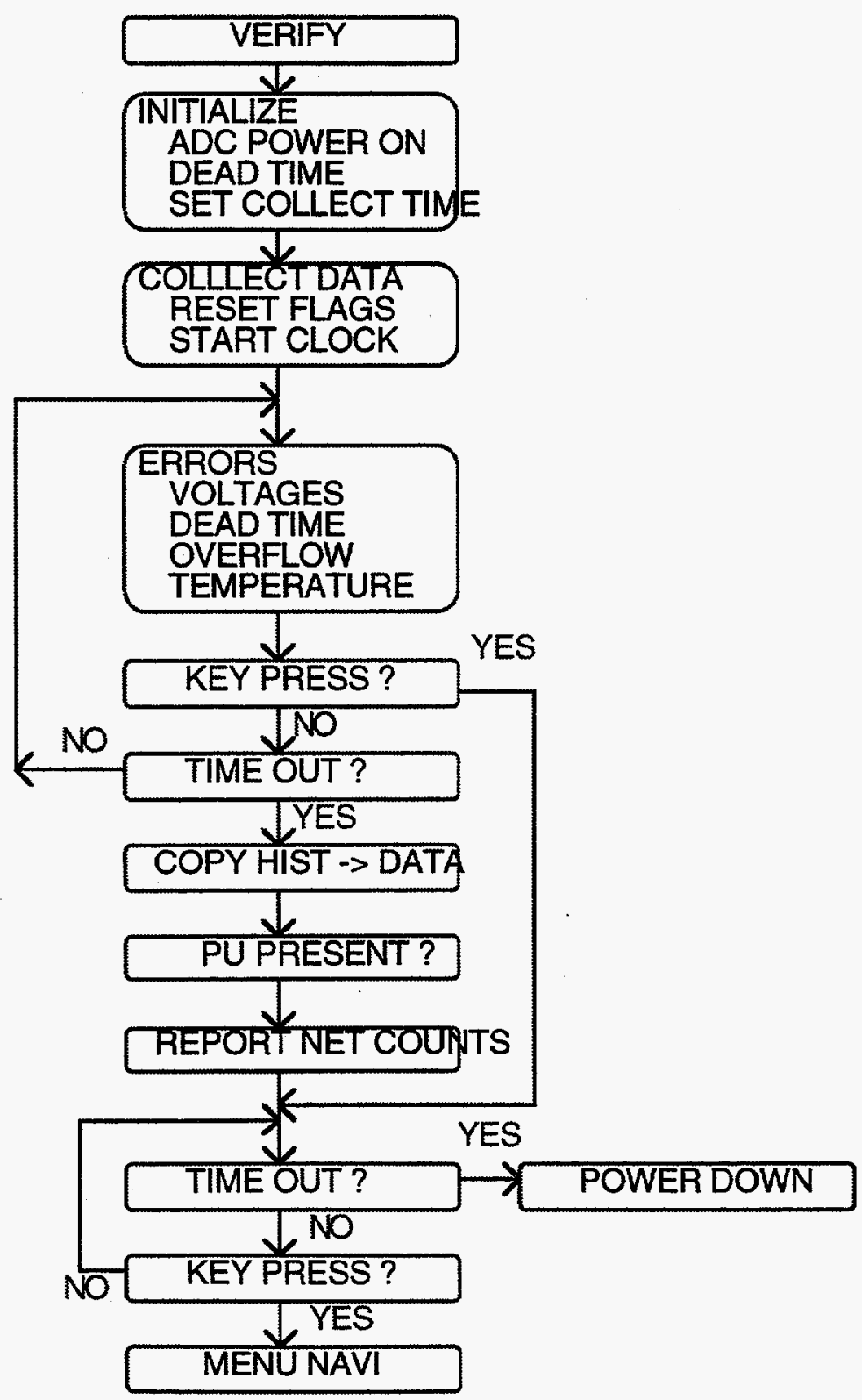

FLOW DIAGRAM 3B 


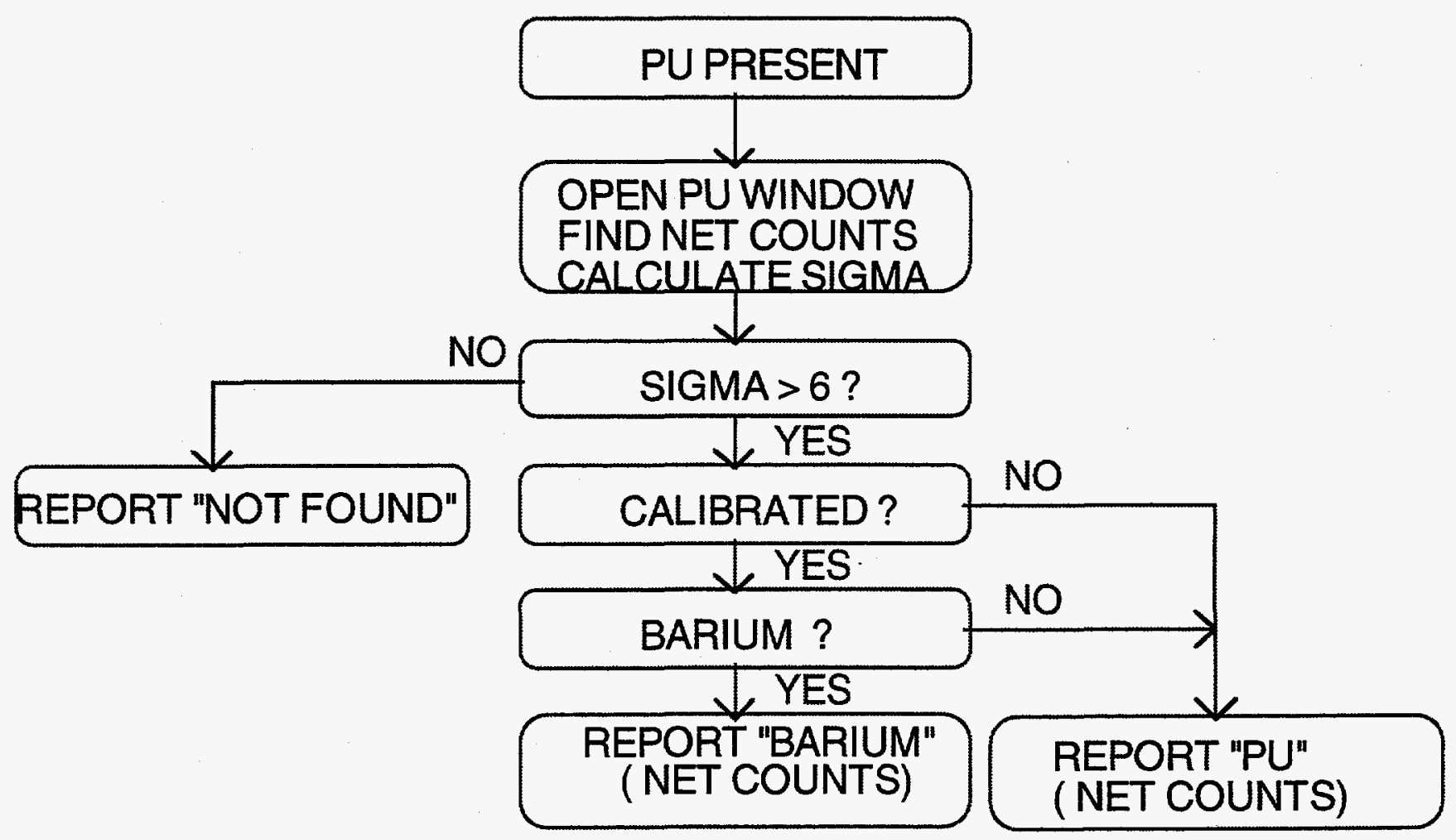

FLOW DIAGRAM 4 


\section{Appendix B. Electrical and Mechanical Specifications Summary}

Gamma Detector

- Bicron NaI(Tl) 1.125 in. dia. $\mathrm{x}$ 2 in. Super- $G$ integrated assy.

- $3^{\circ} \mathrm{C} / \mathrm{min}$ thermal rate of change

- weight $=1.66 \mathrm{lb}$

Removable tungsten collimator

- 3/16 in. thick

- 80\% atten@ $400 \mathrm{keV}$

- $45^{\circ}$ acceptance angle

- weight $=1.78 \mathrm{lbs}$.

\section{Mechanical Package}

- Anodized $6061 \mathrm{Al}$ alloy extrusion

- Machined $\mathrm{Al}$ ends

- NEMA 4 seals

- Machined nose/o-ring seal

- Battery access door

- Membrane keypad with 468MP adhesive

- $1 \mathrm{~atm}$ pressure leak tested

- $\quad$ weight $=2.72 \mathrm{lbs}$.

- total weight $\mathrm{w} /$ collimator $=$ $8.13 \mathrm{lbs}$. $\underline{\text { Neutron Detector }}$

- Reuter-Stokes matched pair He-3, 20 atm. PC

- 0.5 in. dia. $\times 4$ in. active length

- 0.5 in. polyethylene moderator

- non-hazardous gas volume

- weight $=0.42 \mathrm{lbs}$.

\section{Calibration}

\section{Gamma}

- Full Scale Energy $=1.6 \mathrm{MeV}$

- $L L D=60 \mathrm{keV}$

- 3-point quadratic fit using: 80,570 , and $1064 \mathrm{keV}$

Neutron

- must pass gamma disc. test

\section{Electrical Package}

- Batteries: 62/3A lithium

- Operate time: 16 hours

- single button push function keys

- 2 line $x 16$ char. STN backlit LCD

- Microcomputer control

- Non-volatile RAM

- temperature range: -10 to $40^{\circ} \mathrm{C}$

- $\quad$ weight $=1.55 \mathrm{lbs}$.

\section{Pulse Height Analyzer}

- 8-bit FADC/256 channels

- Auto-calibration mode

- Programmable ROIs

- Peak stripping

- Background calculations

- Statistical analysis of ROI activity 
This report has been reproduced from the best available copy.

It is available to DOE and DOE contractors from the Office of Scientific and Technical Information,

P.O. Box 62,

Oak Ridge, TN 37831

Prices are available from

(615) 576-8401.

It is available to the public from the

National Technical Information Service,

US Department of Commerce,

5285 Port Royal Rd.,

Springfield, VA 22161. 


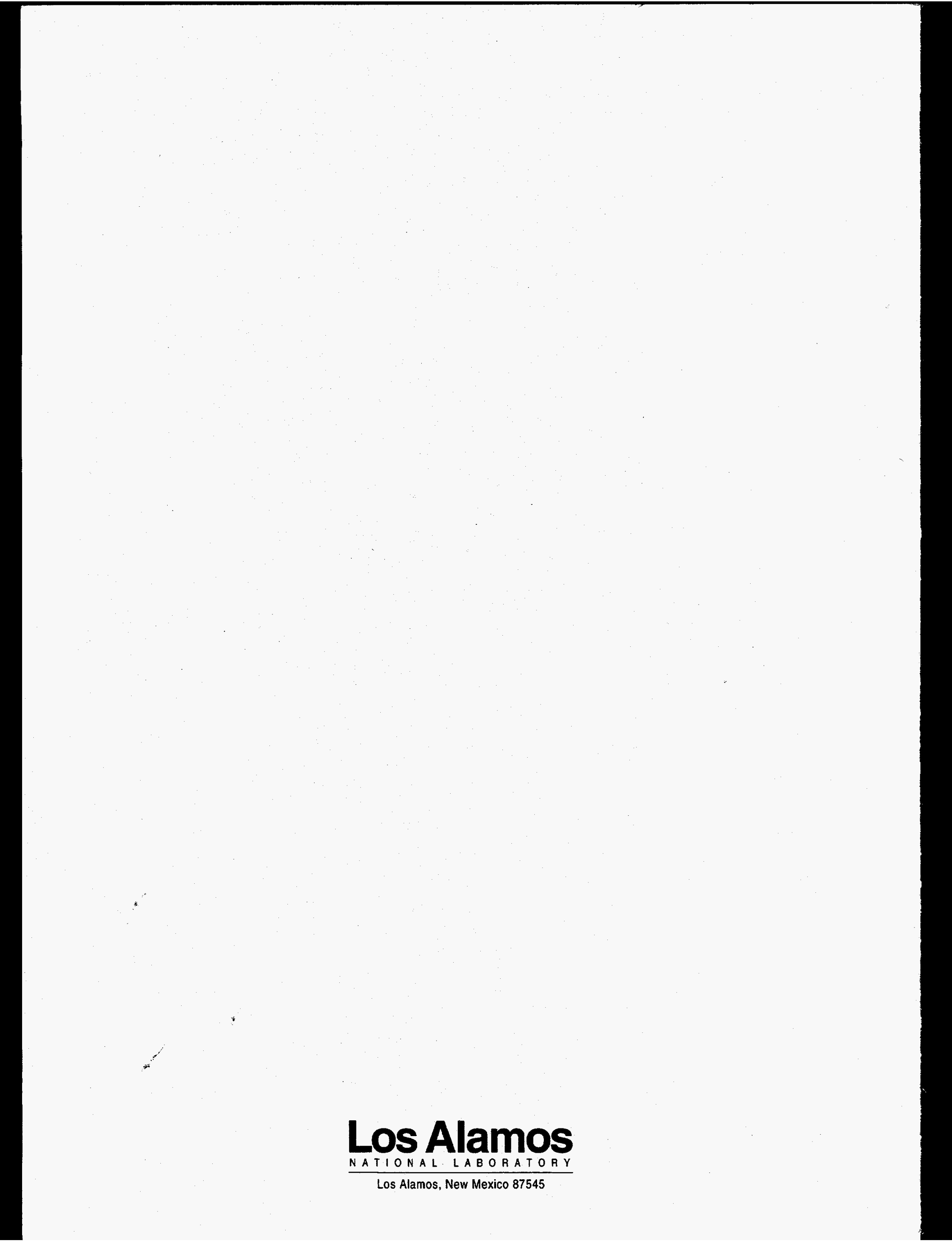

